\title{
Article@Virology
}

\section{The Trend Analysis and Quality Evaluation of Live Attenuated Yellow Fever Vaccine in China from 2011 to 2018}

\author{
Ling Wang, Jingjing Liu, Yan Kong, Yuhua Li* \\ National Institute for Food and Drug Control, Beijing, 102629, P. R. China
}

\begin{abstract}
To summarize the lot release of live attenuated yellow fever vaccine in China during 2011-2018 and evaluate the total quality as well as problem in quality control of the vaccine. By reviewing the data and laboratory test, 34 batches of live attenuated yellow fever vaccine were determined for virus titer according to the standard for license, of which 52 batches were determined for ovalbumin residue by ELISA, and 14 batches for moisture. The determination results were subjected to statistical and quality trend analyses, and compared with those by the manufacturers, based on which the general status of vaccine quality was reviewed. All the determination results of virus titer, ovalbumin residue and moisture met the requirements in Chinese Pharmacopoeia, and there is no difference in those by the manufacturers and those by the National Institutes for Food and Drug Control. The quality of live attenuated yellow fever vaccine was stable, which met the requirements in Chinese Pharmacopoeia. The implementation of lot release, batch inspection and trend analysis may effectively regulate and supervise the vaccine production.
\end{abstract}

Copyright@2012-2020 Published by Hong Kong Institute of Biologicals Standardization Limited. All rights reserved.

Article history: Submitted: 10/07/2019; Revised: 30/07/2019; Accepted: 07/08/2019 DOI: $10.21092 /$ jav.v8i3.113

Key Words: Live attenuated yellow fever vaccine; Quality control; Trend analysis.

Abbreviations: NIFDC, National Institutes for Food and Drug Control; WHO, World Health Organization; SD, standard deviation; ELISA, Enzyme Linked Immunosorbent Assay; PFU, Plaque Forming Unit.

\footnotetext{
* Corresponding author, Major in Viral vaccine quality research F-mail: liyuhua@nifdc.org.cn
} 


\section{Introduction}

Lot release is one of the six basic functions of national management of biological products proposed by the World Health Organization (WHO). In the lot release guidelines, WHO clearly requires that manufacturers and national regulatory authorities/national quality control laboratories should do trend analysis of all key quality control parameters, especially the titer ${ }^{[1]}$. Therefore, trend analysis is an important means of controlling and ensuring product quality consistency, and is also evaluated as a key issue in WHO's pre-certification of vaccine systems ${ }^{[2]}$.

China began to gradually implement the national approval of biological products in 2006, and covered live attenuated yellow fever vaccine in 2010. In this paper, the trend analysis was used to evaluate the quality of the live attenuated yellow fever vaccine from 2011 to 2018, in which the mean and standard deviation (SD) were determined from the test results of National Institutes for Food and Drug Control (NIFDC), and with mean \pm 2 SD as alert, \pm 3 $\mathrm{SD}$ as action ${ }^{[3]}$. Results from the National Institute for Food and Drug Control (NIFDC) and the manufacture were compared to detect the difference of the two Labs.

\section{Materials and methods}

1. Vaccine

105 batches of live attenuated yellow fever vaccine were registered for Lot release from 2011 to 2018. Live attenuated yellow fever vaccines were all from Beijing Biological Products Institute Co., LTD.

\section{Trend analysis of virus titer}

The virus titers of 34 batches were determined by a plaque assay based on the pharmacopoeia method [4]. The titers of these 34 batches from NIFDC were summarized and the trend was analyzed.

3. Trend analysis of Ovalbumin residue

Ovalbumin residues in 52 batches of vaccines were detected by enzyme linked immunosorbent assay (ELISA). Serazym ${ }^{\circledR}$ Ovalbumin e041-a was used. Through the process improvement, amount of ovalbumin residue decreased significantly until the stable stage. Therefore, the average value and SD were calculated by 40 batches of test results after the process improvement, and the trend analysis was conducted.

\section{Trend analysis of Moisture}

Moisture of 14 batches was determined by Fisher's capacity method. The results were summarized and the trend analysis was conducted.

\section{Statistical analysis}

The mean or the geometric mean, the standard deviation (SD) were calculated based on the data from each assay. Alert line in trend analysis is two SD distance from the mean or the geometric mean. Action line in trend analysis is three SD distance from the mean or the geometric 
mean. If the warning line or action line exceeds the standard line, the standard line shall prevail automatically.

\section{Results}

\section{Trend analysis of virus titer}

The geometric mean of virus titer is $5.22 \mathrm{LgPFU} / \mathrm{ml}$, and $\mathrm{SD}$ is $0.33 \mathrm{LgPFU} / \mathrm{ml}$. So, the upper alert line was $5.88 \mathrm{LgPFU} / \mathrm{ml}$, and the lower was $4.56 \mathrm{LgPFU} / \mathrm{ml}$. The virus titer of all samples meets the quality standard, and all test results were within the range of alert line, showing a stable fluctuation trend, as shown in figure 1.

The virus titer of the same batch from NIFDC and enterprises was compared, and the result showed that the virus titer from the two laboratories were in good consistency, as shown in figure 2 .

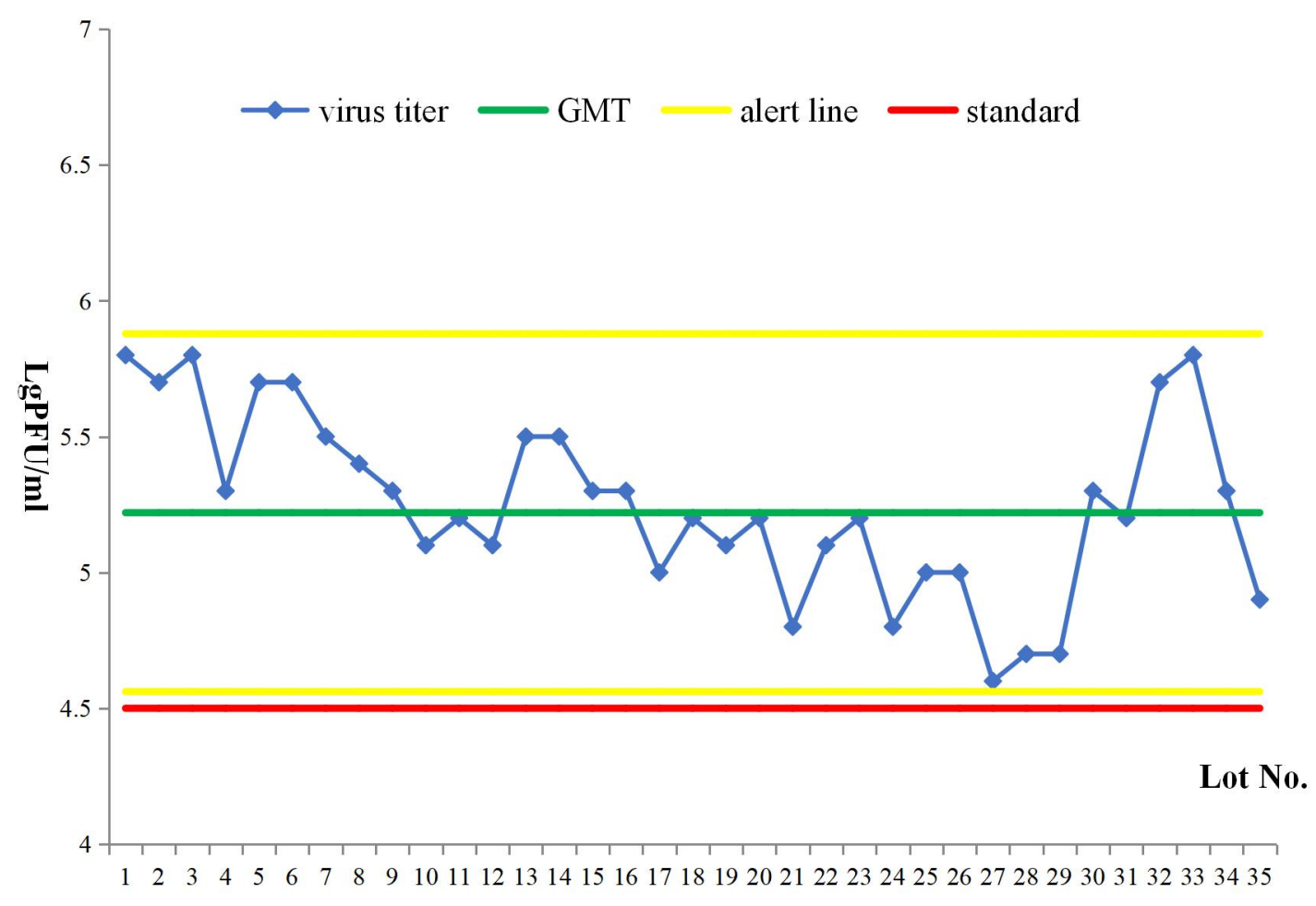

Figure 1: Trend analysis of virus titer based on the data from the NIFDC. 


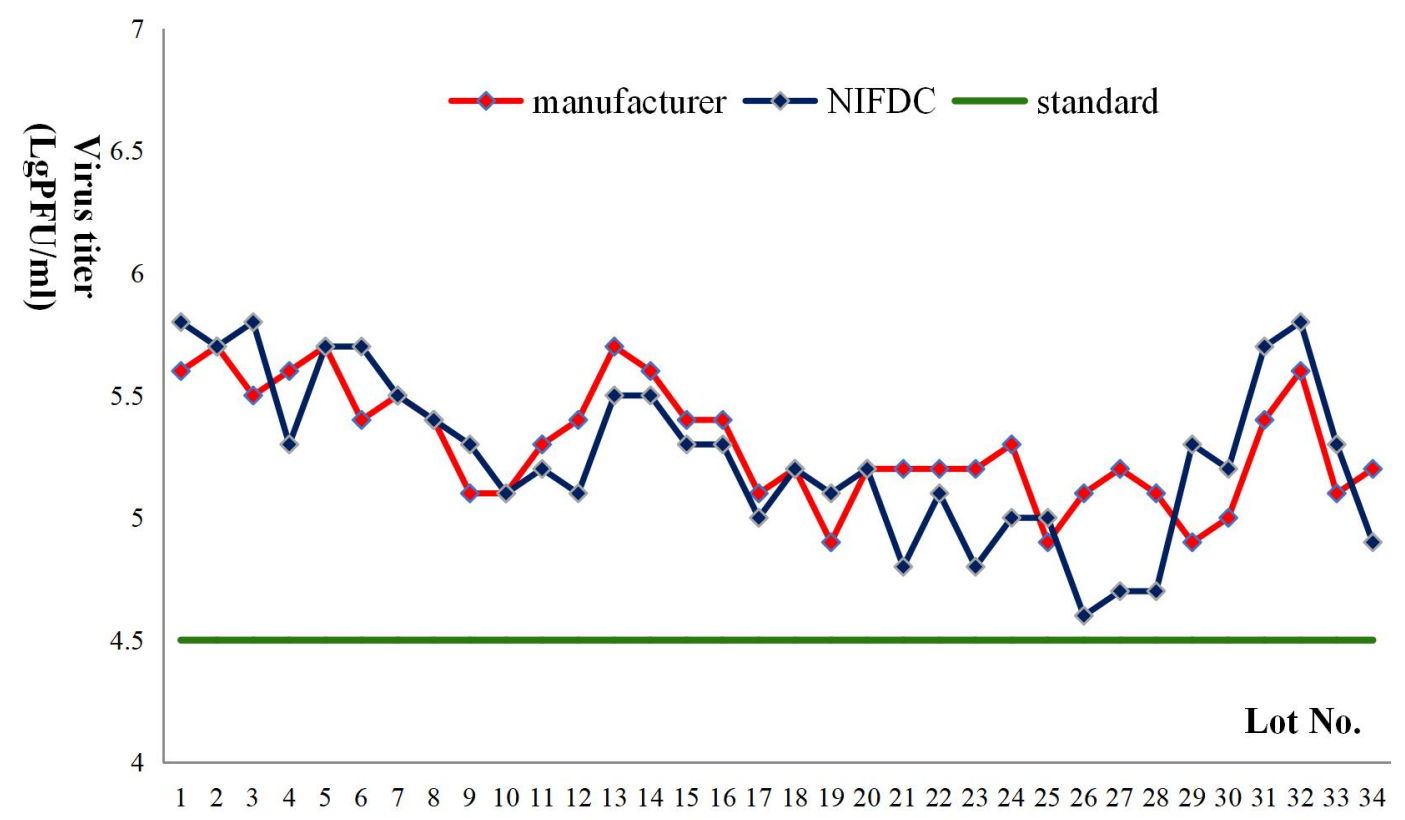

Figure 2: Comparison of the virus titer between NIFDC and those of the enterprise

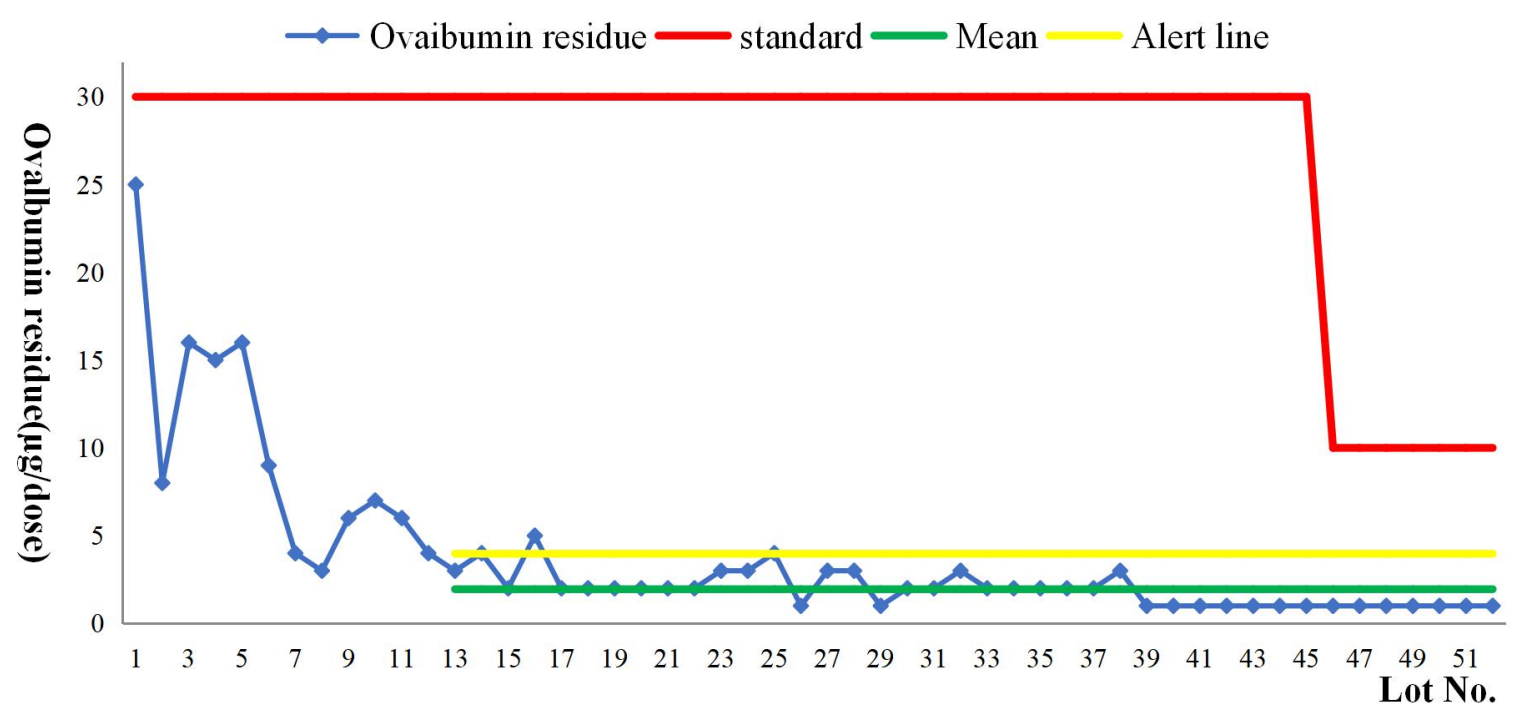

Figure 3: Trend analysis of Ovalbumin residues of live attenuated yellow fever vaccine 
2. Trend analysis of ovalbumin residue

The mean of ovalbumin residue in 52 batches of live attenuated yellow fever vaccine was $1.95 \mu \mathrm{g} / \mathrm{dose}$, and $\mathrm{SD}$ is $1.01 \mu \mathrm{g} / \mathrm{dose}$. So, the upper alert line was $3.97 \mu \mathrm{g} / \mathrm{dose}$, and the lower was less than 0 . All samples conformed to quality standard. Some of data in the earlier batches exceeded the alert line, but that were all far below the quality standard. The ovalbumin residues of nearly 30 batches of vaccines were all within the range of $2 \mathrm{SD}$, showing a stable fluctuation trend, as shown in figure 3.

The Ovalbumin residue of the same batch from NIFDC and enterprises was compared and the result showed that the ovalbumin residue from the two laboratories were in good consistency, as shown in figure 4.

3. Trend analysis of Moisture

The mean of moisture in 14 batches of live attenuated yellow fever vaccine was $1.2 \%$, and $\mathrm{SD}$ is $0.65 \%$. So, the upper alert line was $2.5 \%$, and the lower was less than 0. All samples conformed to quality standard. The moisture of these 15 batches of vaccines were all within the range of $2 \mathrm{SD}$, showing a stable fluctuation trend, as shown in figure 5 .

The comparative analysis of moisture data from NIFDC and enterprises showed that moisture of the two laboratories were in good consistency, as shown in figure 6 .

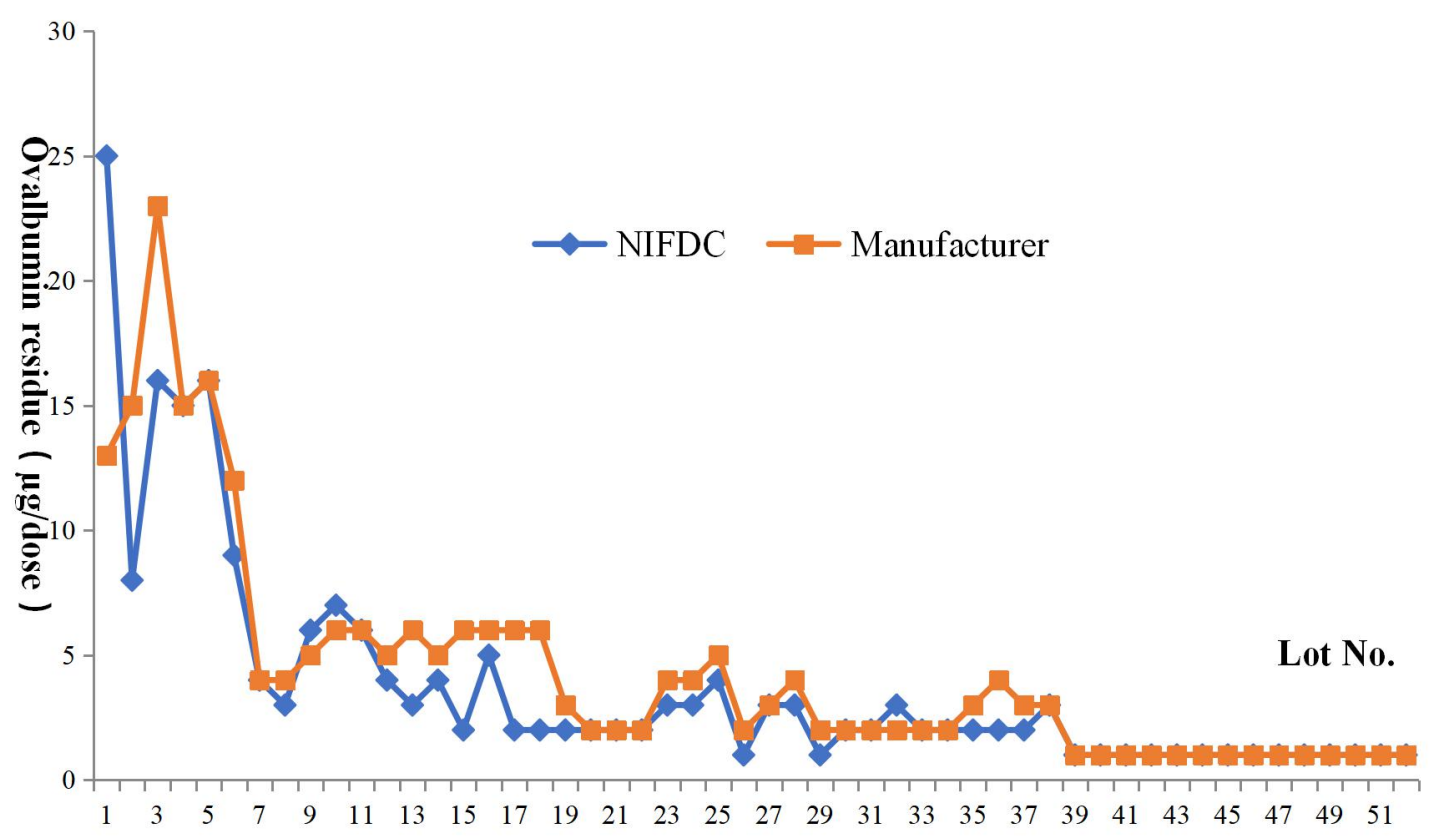

Figure 4: Comparison of the ovalbumin residue between NIFDC and those of the enterprise 


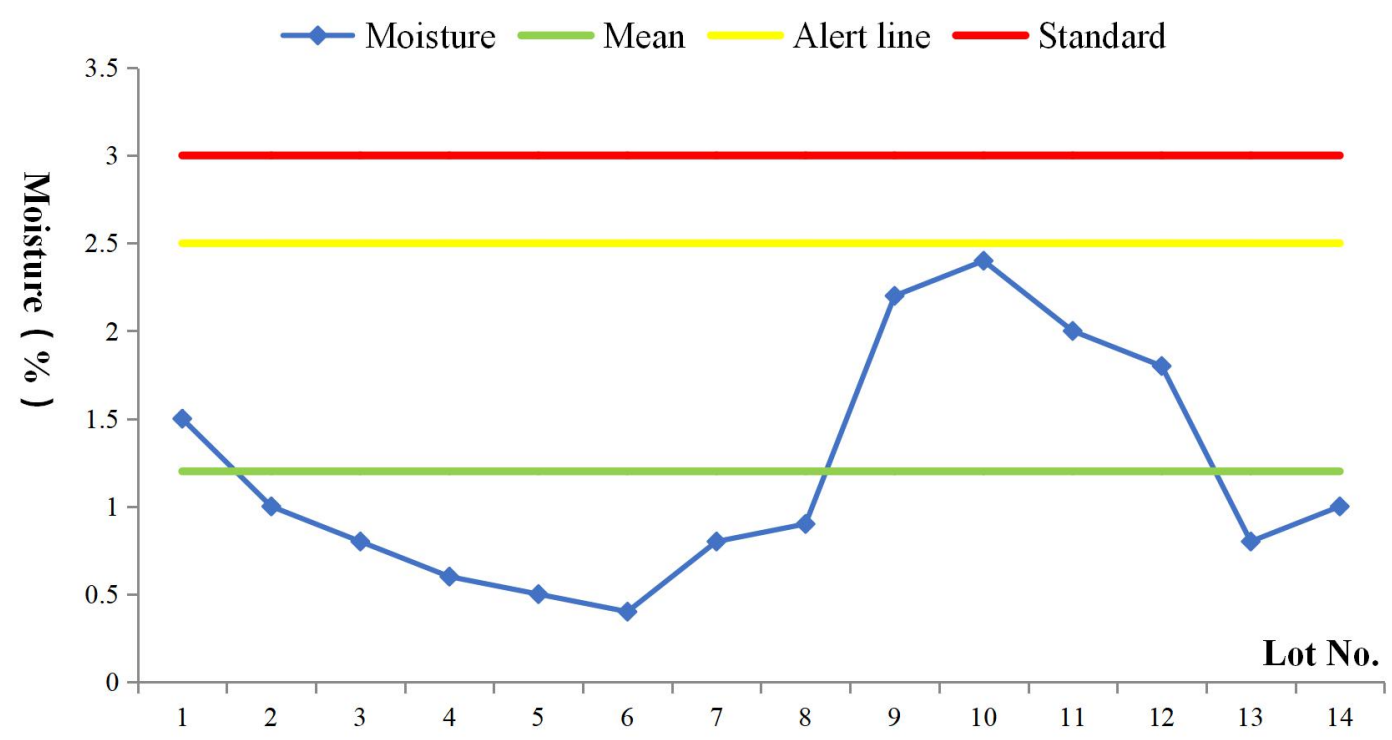

Figure 5: Trend analysis of Ovalbumin residues of live attenuated yellow fever vaccine

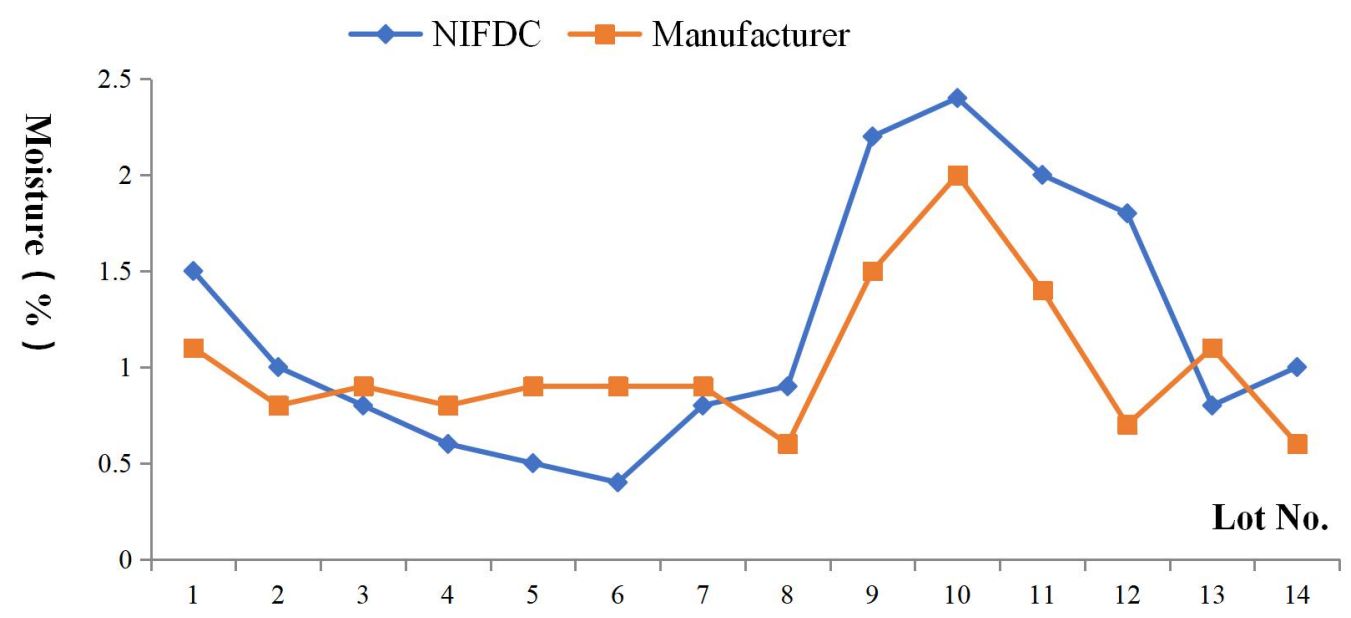

Figure 6: Comparison of the moisure between NIFDC and those of the enterprise. 


\section{Discussion}

Yellow fever vaccination is the most cost-effective preventive measure for yellow fever, especially for controlling yellow fever epidemics in endemic areas and preventing infection among travellers. Although China is not an endemic region for yellow fever, imported yellow fever cases appeared in China for the first time in 2016, with the development of economy, trade and the increase of personnel mobility ${ }^{[5]}$. There is only one vaccine manufacturer for live attenuated yellow fever vaccine in China, with an annual output of about 300,000 doses. The live attenuated yellow fever vaccine produced in China is mainly used for people who are going to pass or arrive at the yellow fever epidemic area.

It has been 9 years since the live attenuated yellow fever vaccine was licensed of Lot Release. Lot release was based on the examination of summary manufacturing protocol and laboratory tests. However, the national quality control laboratory has paid more and more attention to the trend analysis of test results in recent years. Trend analysis is an important part of WHO's Lot release guidelines ${ }^{[6]}$. Trend analysis plays an important role in quality control of vaccines. Through the trend analysis of the verification results, the consistency between batches of vaccines can be monitored, thus indirectly reflecting the stability of the vaccine production process. Only by ensuring the quality consistency of vaccines from batch to batch, can the representativeness of clinical trials be reflected, so as to ensure the safety and effectiveness of each batch of vaccines when used in human.

Since the lot release of live attenuated yellow fever vaccine from 2010, 105 batches have been issued, with more than 3 million doses. The qualified rate was $100 \%$. Through trend analysis of the virus titer, ovalbumin residue and moisture of the vaccine, the results show that the quality of the live attenuated yellow fever vaccine is stable and the consistency between batches is good. All test results are within the alert line. And, the comparison of test data between NIFDC and enterprises shows that the results of the two laboratories are consistent. On the whole, the production process and quality control method of live attenuated yellow fever vaccine in China are stable. These also guaranteed the quality stability of live attenuated yellow fever vaccine in China.

\section{References}

[1]. WHO. Guidelines for independent lot release of vaccines by regulatory authorities[S/OL]. http://www.who.int/biologicals/Guidelines for Lot Release AFTER ECBS
27.1.2011.pdf

[2]. Liu XY, Zhang J, Jia LL, et al. Application of trend analysis in quality control of live attenuated Japanese encephalitis vaccine[J]. Chin J Biologicals, 2012, 25(11):1562-1564. 
[3]. Ma X, Meng L, Tan YJ, et al. Application of trend analysis method for vaccine lot release [J]. Chin Pharm Affa,2014,28(7):750-753.

[4]. Chinese Pharmacopoeia Commission. Pharma-copoeia of People's Republic of China( first enlarged edition)[S]. Beijing: China Med Sci Press, 2018:323-325.

[5]. Chambers TJ, Nickells M. Neuroadapted yellow fever virus 17D: genetic and biological characterization of a highly mouse-neurovirulent virus and its infectious molecular clone[J]. J Virol, 2001,75(22): 10912-10922.

[6]. WHO Expert Committee on Biological Standardization. Guidelines for independent lot release of vaccines by regulatory authorities [S]. 2010-10-18. 